\title{
Estimación de la Tasa de Deshidratación Foliar y su Importancia para la Gestión de Ecosistemas Terrestres en el Caribe Colombiano
}

\section{Foliar Dehydration Rate Estimation and its Importance for the Terrestrial Ecosystems in the Caribbean Region of Colombia}

\author{
Juan Carlos Valdelamar Villegas ${ }^{1}$, Katherine Prada Sánchez ${ }^{2 a}$, Karla Gamarra Cabarcas ${ }^{2 b}$ \\ ${ }_{1}^{1}$ Docente titular, Departamento de Ingeniería Ambiental, Grupo de Investigación Ambiental (GIA), Fundación Universitaria \\ Tecnológico Comfenalco, Sede A, Barrio España, Cra. 44 D No. 30 A-91, Cartagena, Colombia. \\ jvaldelamar@tecnologicocomfenalco.edu.co \\ 2 Estudiante de Ingeniería Ambiental, Departamento de Ingeniería Ambiental, Grupo de Investigación Ambiental (GIA), \\ Fundación Universitaria Tecnológico Comfenalco, Sede A, Barrio España, Cra. 44 D No. 30 A-91, Cartagena, Colombia. \\ a ktmarps@gmail.com \\ b karlys219@gmail.com
}

Recibido: 20Ago2014 - Revisado: 11Sep2014

Aceptado: 30Oct2014 - Publicado: 10Dic2014

\begin{abstract}
Resumen: El conocimiento de la biología de las plantas, especialmente de la tolerancia que estas pueden tener frente a las actuales alteraciones ambientales en lo que hace referencia al cambio climático, es de gran importancia para identificar aquellas especies capaces de resistir situaciones de estrés hídrico que se producen en las plantas debido al aumento de su tasa de evapotranspiración y deshidratación. Por ello, mediante el presente estudio, se comparó la tasa de deshidratación foliar en once de las principales especies de plantas presentes en dos ecosistemas estratégicos de Cartagena, Colombia (caño Juan Angola y cerro de La Popa), los cuales pertenecen en su mayoría (36\%) a la familia Fabaceae, seguida de las Malvaceae, Rubiaceae, Ancaradiaceae, Sapindaceae, Combrataceae, Apocynaceae y la Rizophoraceae, con 9\% cada una. Los resultados ubican a la especie Laguncularia racemosa como la de mayor tasa de deshidratación foliar $\left(23,35 \pm \mathrm{mg} \mathrm{H}_{2} \mathrm{O} / \mathrm{hr}\right)$; mientras que Guazima ulmifolia fue la especie con menor tasa de deshidratación $(3,51 \pm 1,63 \mathrm{mgH}, \mathrm{O} / \mathrm{hr})$. Se discuten los resultados encontrados en el estudio, con respecto a la importancia de la información obtenida para la gestión ecológica y ambiental de los ecosistemas locales de la ciudad ante un futuro escenario de cambio climático.
\end{abstract}

Palabras claves: Cambio climático, deshidratación foliar, evapotranspiración, temperatura, balance hídrico.

Abstract: The knowledge of the plants biology, especially of the tolerance these may have against current environmental changes with respect to climate change, is of a great importance to identify those species able to resist water stress produced due to both increased evapotranspiration rate and dehydration. Therefore, by means of this study, the rates of leaf dehydration of eleven major plant species were compared. These species are mainly present within two strategic ecosystems of Cartagena, Colombia (Juan Angola creek and La Popa hill), which belong mostly (36\%) to the Fabaceae family, followed by Malvaceae, Rubiaceae, Ancaradiaceae, Sapindaceae, Combrataceae, Apocynaceae, and Rizophoraceae with 9\% each. The results place the Laguncularia racemosa species as the one with the highest rate of leaf dehydration $\left(23.35 \pm \mathrm{mg} \mathrm{H}_{2} \mathrm{O} / \mathrm{h}\right)$; while Guazima ulmifolia was the species with the lowest rate of dehydration $\left(3.51 \pm 1.63 \mathrm{mg} \mathrm{H}_{2} \mathrm{O} / \mathrm{h}\right)$. The results found in the study are discussed with respect to the importance of the information obtained for ecological and environmental management of local ecosystems of the city in the context of a future scenario of climate change.

Key words: Climate change, foliar dehydration, evapotranspiration, temperature, water balance.

\section{INTRODUCCIÓN}

Para sobrevivir y desarrollarse de forma normal, las plantas perciben constantemente cambios en el ambiente circundante y responden a través de una variedad de mecanismos que a su vez le ayudan a prestar servicios ecosistémicos como provisión de alimentos y materia prima, cobertura vegetal, regulación del microclima, regulación de gases $\left(\mathrm{CO}_{2}\right.$ y $\left.\mathrm{O}_{2}\right)$, protección de los suelos contra la erosión y balance hídrico, entre otras.

Actualmente, los principales problemas que afrontan los ecosistemas terrestres son la tala indiscriminada, contaminación química, introducción de especies foráneas, alteración de las condiciones climáticas y pérdida de la biodiversidad asociada (Minambiente, 2014). Estas problemáticas pueden verse relacionadas por el desconocimiento de algunos factores 
como la pérdida de humedad por evaporación directa junto con la pérdida de agua por transpiración, ya que este es un factor de vital importancia al momento de analizar las causas de afectación a la vegetación de un ecosistema. Esto puede analizarse en las hojas de las plantas. La función esencial de estas está en el mantenimiento de la homeostasis de la planta, especialmente en la regulación fisiológica de la pérdida de vapor de agua e ingreso de dióxido de carbono (Toldi et al., 2009). El intercambio de estos y otros gases en las hojas se realizan por medio de los estomas, que constituyen el mecanismo biológico más importante de la planta predominando su función en la transpiración y la fotosíntesis (Jones, 1995). También son el órgano más susceptible en responder a las condiciones del medio ambiente y es capaz de reflejar más claramente, los efectos de estrés impuestos por factores ambientales o a su heterogeneidad (Bartoskova et al., 1999; Zegbe \& Hossein, 2010).

La mayoría de estudios relacionados con el análisis de la pérdida de humedad en las plantas está enfocado solamente para los cultivos, ya que por medio de esto se permite ajustar las dosis de riego como los momentos de aplicación, logrando de esta manera un uso eficiente del agua de riego, ahorrando agua y energía y mejorando a su vez la rentabilidad de los agricultores y aumentando la sustentabilidad en las prácticas de riego en épocas de sequía (Basnayake, 1996; Pence, 2005; Toldi et al., 2009; Oliver et al., 2011).
El uso eficiente de agua por las plantas terrestres favorece el balance energético de la hoja, ya que el agua evaporada desde las células del mesófilo enfría las superficies foliares (González-Bermúdez, 2013). La corriente transpiratoria favorece el transporte de nutrientes inorgánicos procedentes del suelo y el transporte de solutos orgánicos, aminoácidos y fitohormonas desde la raíz a los órganos de transpiración (RodríguezPérez, 2006). Por tal razón, el objetivo de esta investigación es determinar la tasa de deshidratación foliar de las diferentes especies de dos ecosistemas estratégicos de la ciudad de Cartagena de Indias (el cerro de La Popa y caño Juan Angola); además de su importancia para la gestión de ecosistemas terrestres como medida a tener en cuenta cuando se pretenda realizar reforestación en un proceso de conservación.

\section{MATERIALES Y MÉTODOS}

\section{1 Área de estudio}

El presente estudio se desarrolló en dos ecosistemas estratégicos de la ciudad de Cartagena de Indias, el cerro de La Popa (10 $25^{\prime} 20,94^{\prime \prime} \mathrm{N}-7^{\circ} 31^{\prime} 38,39^{\prime \prime}$ O) y caño

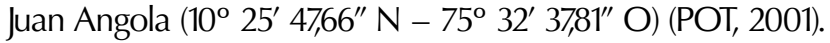
La zona de estudio (Figura 1) se caracteriza por presentar dos periodos climáticos: uno lluvioso comprendido entre los

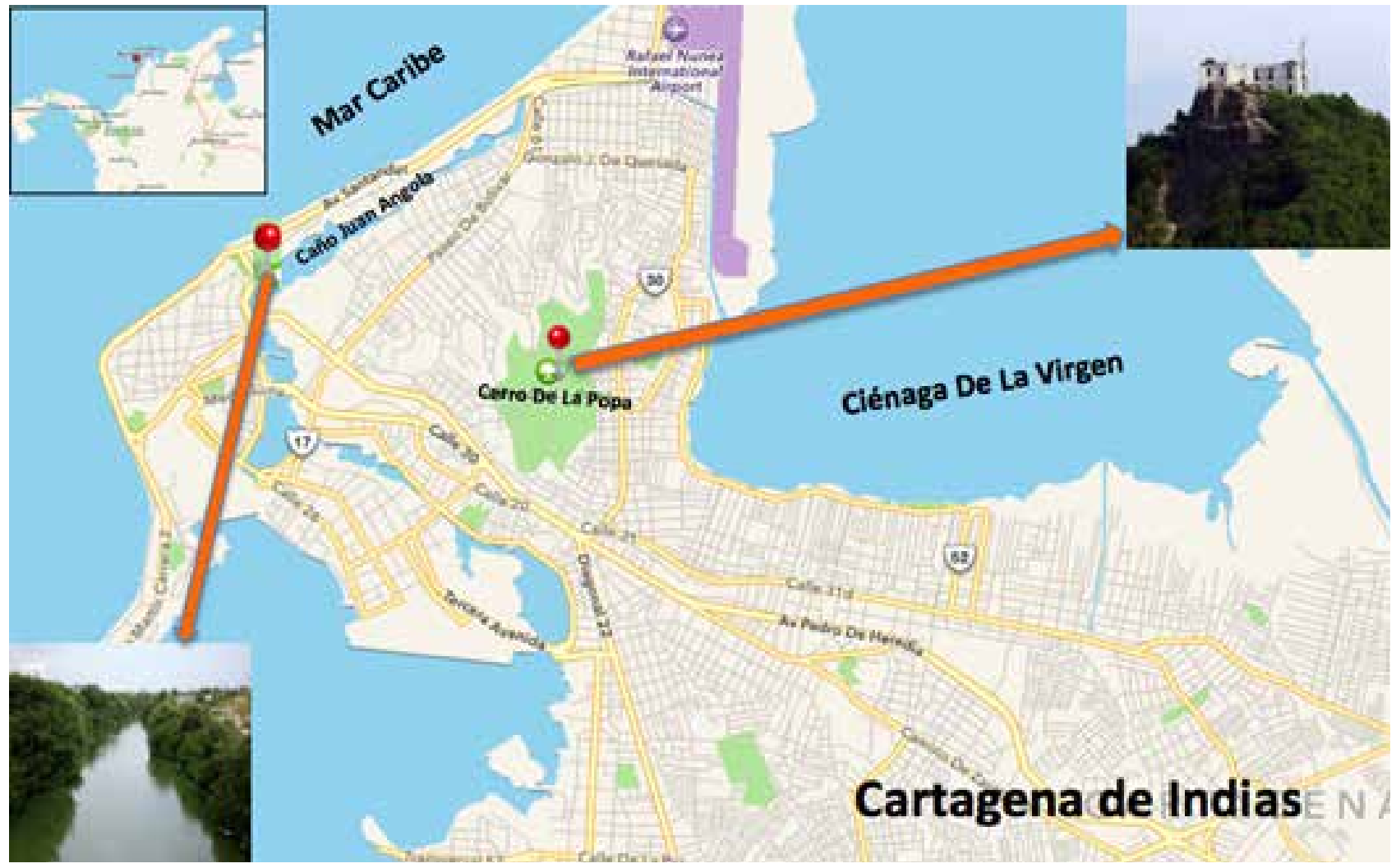

Fig. 1. Ubicación del cerro de La Popa y el caño Juan Angola en la ciudad de Cartagena, caribe colombiano. Tomado y modificado de Google Maps. 


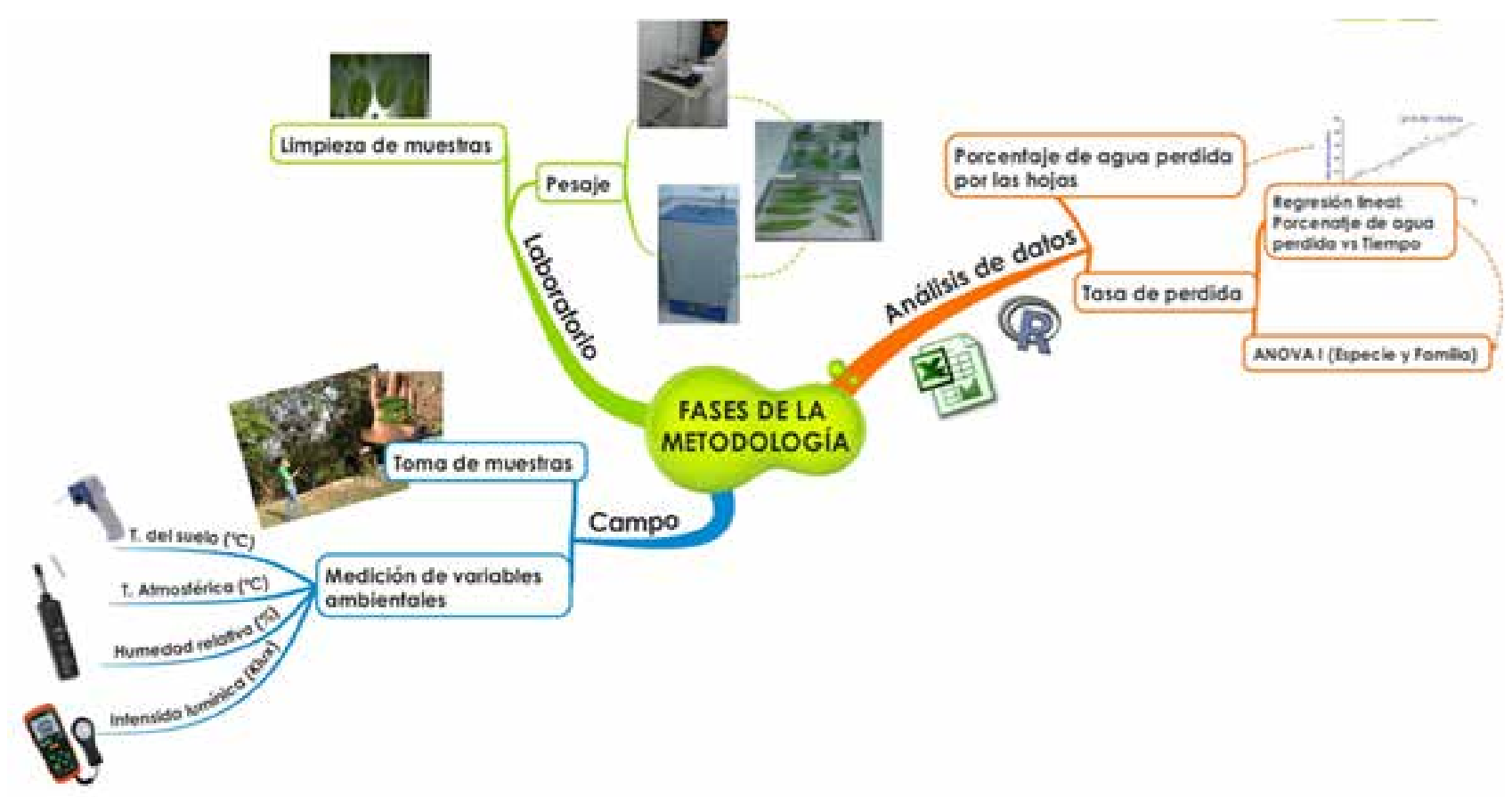

Fig. 2. Aspectos metodológicos contemplados durante el estudio.

meses de abril y noviembre y el otro periodo se denomina, seco y se presenta desde el mes de diciembre, hasta abril. Sin embargo, también existe un periodo de transición entre los meses junio y julio, conocido como veranillo de San Juan. El promedio anual de la precipitación es de $51,4 \mathrm{~mm}$ con una temperatura que oscila entre los $28^{\circ} \mathrm{C}$ y $\operatorname{los} 30^{\circ} \mathrm{C}$ (Afanador et al., 2008).

\subsection{Fase de Campo}

En esta fase se realizaron salidas de campo al caño Juan Angola y cerro de La Popa, para la medición de variables las cuales fueron: temperatura del suelo, temperatura atmosférica, humedad relativa e intensidad lumínica y la toma de muestra. Para llevar a cabo esto, se seleccionaron de manera aleatoria varios individuos y se procedió a realizar la colecta de las hojas que fueron introducidas en bolsas Ziploc $®$ y rotuladas, para posteriormente ser trasportadas al laboratorio de ciencias biológicas y realizarles caracterización biométrica y estimación del porcentaje de agua pérdida por las hojas.

\subsection{Fase de laboratorio}

En esta fase se realizó la limpieza de las muestras de hoja y su pesaje en una balanza analítica de precisión en $\mathrm{mg}^{-1}$ (Garcés \& Forcelini, 2011). Posteriormente, las hojas se introdujeron en un horno automático graduado con temperatura igual a $40^{\circ} \mathrm{C}$, del cual se extraían, pesaban y regresaban las hojas de forma reiterada cada 20 minutos. El proceso se repitió hasta que las muestras mantuvieron un peso constante.

\section{4 Procesamiento y análisis de datos}

La tasa de deshidratación foliar $\left(\mathrm{mgH}_{2} \mathrm{O} / \mathrm{h}\right)$ para cada especie, se halló mediante el cálculo de la pendiente de la recta resultante de la regresión lineal efectuada entre el tiempo (variable independiente) y la pérdida de agua (variable dependiente), para cada regresión solo se contemplaron valores del coeficiente de determinación $\left(R^{2}\right)$ superiores al $90 \%$. Los datos colectados se tabularon para posteriormente realizar los cálculos estadísticos descriptivos y comparar la tasa de deshidratación foliar para cada especie y familia, mediante un análisis de varianza (ANOVA). La totalidad de los análisis estadísticos se hizo con ayuda el paquete estadístico R 3.0.2.

Las fases relacionadas con las actividades de campo y laboratorios se resumen en la Figura 2.

\section{RESULTADOS}

\subsection{Características meteorológicas del área de estudio}

Como resultado de las mediaciones efectuadas de las variables meteorológicas (Tabla 1), en los dos sitios de estudio pudo apreciarse que el cerro de La Popa se diferencia del caño Juan Angola por sus mayores valores de temperatura del suelo $\left(32^{\circ} \mathrm{C}\right.$ ) e intensidad lumínica (44 Klux) que logra llegar al suelo. Sin embargo, al analizar la humedad relativa, como era de esperarse, el caño Juan Angola presentó los mayores valores por el efecto de la evaporación del agua presente en dicho ecosistema. A pesar de ello, la temperatura atmosférica no mostró gran diferencia entre los ecosistemas. 
Tabla 1. Valores promedios de las variables meteorológicas medidas en el cerro de la Popa y el caño Juan Angola. Cartagena de Indias.

\begin{tabular}{|c|c|c|c|c|}
\cline { 2 - 5 } \multicolumn{1}{c|}{} & \multicolumn{2}{c|}{ Cerro de La Popa } & \multicolumn{2}{c|}{ Caño Juan Angola } \\
\hline Variable & Promedio & $\begin{array}{c}\text { Desv. } \\
\text { estándar }\end{array}$ & Promedio & $\begin{array}{c}\text { Desv. } \\
\text { estándar }\end{array}$ \\
\hline Temp. de suelo $\left({ }^{\circ} \mathrm{C}\right)$ & 32,00 & 4,24 & 31,75 & 1,55 \\
\hline Temp. atmosférica $\left({ }^{\circ} \mathrm{C}\right)$ & 24,60 & 0,28 & 24,68 & 0,64 \\
\hline Humedad relativa $(\%)$ & 69,85 & 0,49 & 74,20 & 2,71 \\
\hline Intensidad lumínica (Klux) & 44,00 & 1,41 & 40,67 & 8,65 \\
\hline
\end{tabular}

\subsection{Especies de plantas analizadas}

El presente estudio se llevó a cabo con once especies de plantas de mayor frecuencia en los ecosistemas estudiados, cuya ubicación taxonómica se muestra en la Tabla 2, destacándose entre estas el ciruelo (Spondias purpurea) y el mamón (Melicoccus bijugatus) por su gran aceptación en las comunidades de la costa caribe colombiana dado su carácter frutal; mientras que otras como el guazimo (Guazuma ulmifolia) y el mataratón (Gliricidia sepium), por sus propiedades medicinales. Al analizar la composición de las mismas especies pero agrupadas por familia, se observa como familia dominante a las Fabaceae (37\%), siendo igual la representación porcentual (9\%) para el resto de las familias (Figura 3).

\begin{tabular}{|c|c|c|}
\hline Familia & N. Científico & N. Común \\
\hline Anacardiaceae & Spondias purpurea & Ciruelo \\
\hline Apocynaceae & Calotropis procera & Algodón de playa \\
\hline \multirow{2}{*}{ Combretaceae } & Laguncularia racemosa & Mangle blanco \\
\hline \multirow{2}{*}{ Fabaceae } & Caesalpinia coriaria & Dividivi \\
\cline { 2 - 3 } & Mimosa pudica & Dormidera \\
\cline { 2 - 3 } & Prosopis juliflora & Trupillo \\
\hline Malvaceae & Guazuma ulmifolia & Mataratón \\
\hline Rhizophoraceae & Rhizophora mangle & Mangle rojo \\
\hline Rubiaceae & Morinda royoc L. & Piñita \\
\hline Sapindaceae & Melicoccus bijugatus & Mamón \\
\hline
\end{tabular}

Tabla 2. Especies y familias de plantas del cerro de La Popa y el caño Juan Angola empeladas durante el presente estudio. Cartagena de Indias.
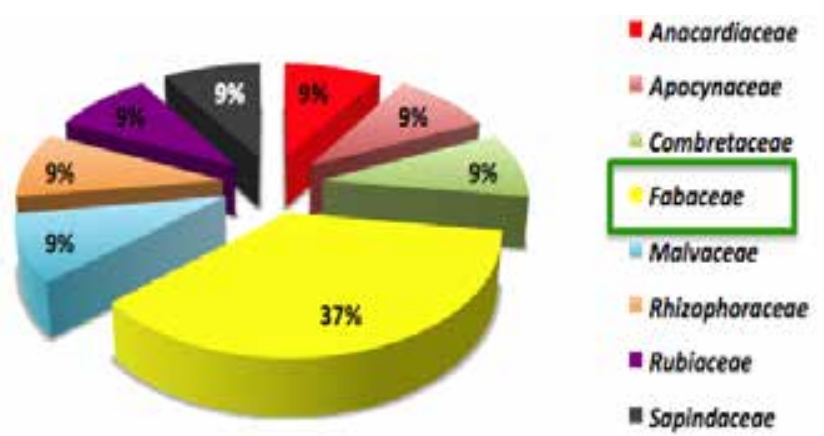

Fig. 3. Distribución porcentual de la frecuencia de familias de plantas del cerro de La Popa y el caño Juan Angola empeladas durante el presente estudio. Cartagena de Indias

\subsection{Comparación de las tasas de deshidratación foliar}

Como resultado de la comparación de las tasas de deshidratación foliar para cada una de las especies analizadas, se apreció que existían diferencias estadísticamente significativas $(P$-valor $<0,05)$ entre estas (Tabla 3 y Figura 4), siendo mayor los valores de esta tasa para las especies $L$. racemosa $\left(23,25 \mathrm{mgH}_{2} \mathrm{O} / \mathrm{h}\right)$ seguida de las especies M. Bijugatus $(15,33$ $\left.\mathrm{mgH}_{2} \mathrm{O} / \mathrm{h}\right), S$. purpurea $\left(14,91 \mathrm{mgH}_{2} \mathrm{O} / \mathrm{h}\right)$, G. Sepium $(14,38$ $\left.\mathrm{mgH}_{2} \mathrm{O} / \mathrm{h}\right), P$. juliflora $\left(13,89 \mathrm{mgH}_{2} \mathrm{O} / \mathrm{h}\right)$ y $C$. procera $(12,53$ $\mathrm{mgH} \mathrm{H}_{2} \mathrm{O} / \mathrm{h}$ ). El resto de especies presentó tasas que se pueden considerar como bajas, debido a que no superaron los $10 \mathrm{mgH} \mathrm{H}_{2} \mathrm{O} / \mathrm{h}$.

Tabla 3. ANOVA para la tasa de deshidratación foliar de las once especies analizadas.
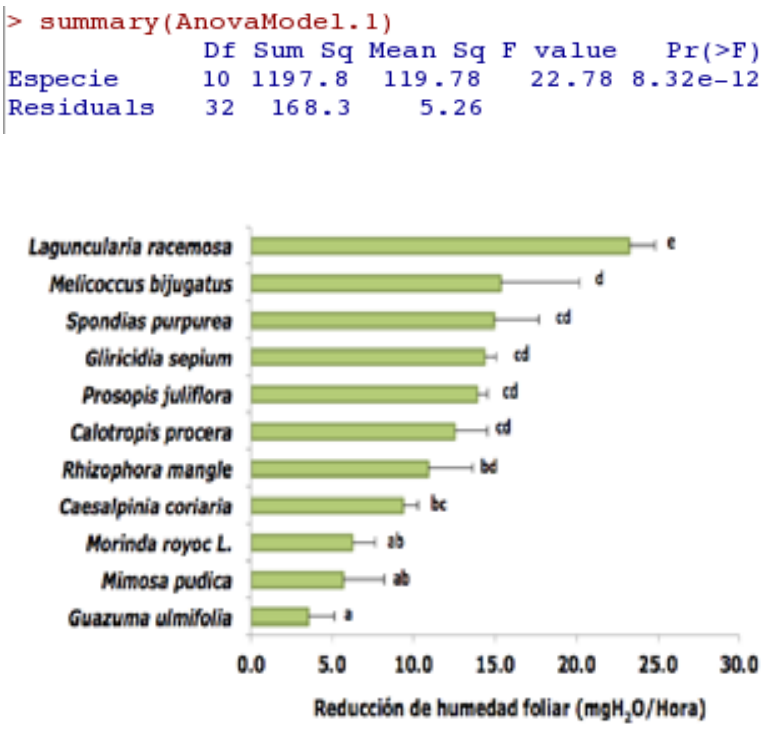

Fig. 4. Tasa de deshidratación foliar promedio, para las especies de plantas presentes en el caño Juan Angola y el cerro de La Popa. Cartagena de Indias. 
El análisis comparativo de las tasas de deshidratación foliar efectuado para las familias de plantas, mostró que también existían diferencias estadísticamente significativas entre estas (P-valor $<0,05$ ) (Tabla 4 y Figura 5), cuyos promedios fueron comparados en la Figura 5, en donde pudo apreciarse que la familia Combretaceae, fue quien presento la mayor tasa de deshidratación foliar $\left(23,2 \mathrm{mgH}_{2} \mathrm{O} / \mathrm{h}\right)$, seguida de la Sapindaceae $\left(15,3 \mathrm{mgH}_{2} \mathrm{O} / \mathrm{h}\right)$, Anacardaceae $\left(14,9 \mathrm{mgH}_{2} \mathrm{O} / \mathrm{h}\right.$, Apocynaceae $\left(12,5 \mathrm{mgH}_{2} \mathrm{O} / \mathrm{h}\right)$ y Fabaceae $\left(11,2 \mathrm{mgH}_{2} \mathrm{O} / \mathrm{h}\right)$; siendo la tasa para el resto de familias inferior a $10 \mathrm{mgH}_{2} \mathrm{O} / \mathrm{h}$.

Tabla 4. ANOVA para la tasa de deshidratación foliar de las ocho familias analizadas.

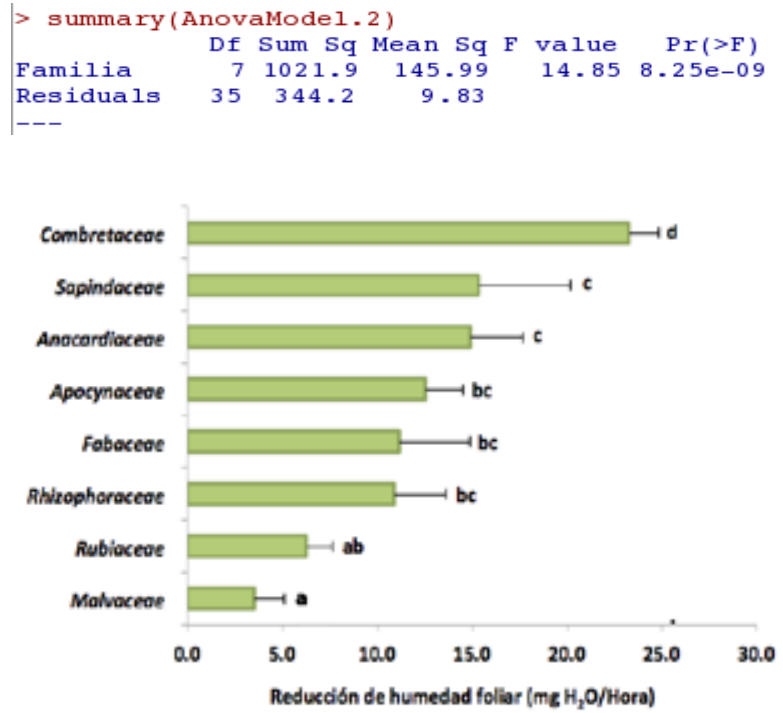

Fig. 5. Tasa de deshidratación foliar promedio, para las familias de plantas presentes en el caño Juan Angola y el cerro de La Popa. Cartagena de Indias.

\section{DISCUSIÓN}

La mayor temperatura del suelo encontrada en el cerro de la Popa, está directamente relacionado con la poca cobertura vegetal que existe en esta zona, lo cual también se hizo evidente por los altos valores de intensidad lumínica que alcanzan a llegar al suelo, como se pudo evidenciar en los resultados, los cuales resultan relevantes, ya que permiten visualizar el papel de la vegetación como elemento de gran importancia para regular el ciclo del recurso agua en los ecosistemas, así como también la forma como esta regula el comportamiento de las variables meteorológicas.

El conocimiento de las características mencionadas en el párrafo anterior resultan relevantes para analizar el comportamiento fisiológico de las plantas (Drennan, 1999), sobre todo en lo que se refiere a la regulación de su contenido de agua, cuyo estudio se puede abarcar mediante tres métodos: muestreo de campo, información meteorológica y teledetección. Los tres sistemas tienen ventajas e inconvenientes. El trabajo de campo resulta el método más directo y preciso, pero tiene el inconveniente de ser costoso y lento, además de contar con un carácter muy local, lo que dificulta su aplicación operativa (Cocero et al., 2000).

El empleo de índices de humedad basados en datos meteorológicos es una práctica común en la estimación de la evapotranspiración o del riesgo de incendios (Camia et al., 1999); sin embargo el uso de métodos rápidos y de baja complejidad para la de estimación de la perdida de humedad de las plantas resulta de gran ayuda en la actualidad, ya que con ello se podría simular el efecto de las variables meteorológicas como la temperatura atmosférica sobre la actividad fisiológica de las plantas (Chuvieco et al., 2001), cuya información resulta de gran importancia para la gestión de los ecosistemas terrestres en estos momentos de incertidumbre sobre el efecto que puede tener el calentamiento global sobre las especies locales, sobre todo en los ecosistemas locales.

Teniendo en cuenta las consideraciones anteriores, así como los resultados hallados durante la presente investigación, se pueden considerar a las familias Malvaceae (3,5 mgH $\left.\mathrm{m}_{2} \mathrm{O} / \mathrm{h}\right)$, Rhizophoraceae $\left(10,9 \mathrm{mgH}_{2} \mathrm{O} / \mathrm{h}\right)$, Rubiaceae $\left(6,2 \mathrm{mgH}_{2} \mathrm{O} / \mathrm{h}\right)$, Fabaceae $\left(11,2 \mathrm{mgH}_{2} \mathrm{O} / \mathrm{h}\right)$, como las de mayor grado de tolerancia a la deshidratación foliar, por incremento en los valores de temperatura atmosférica de los ecosistemas locales en Cartagena de Indias, se destaca especialmente la familia Malvaceae a la que pertenece la especie Guazima ulmifolia, también llamado Guazimo, por ser un árbol de porte pequeño a mediano, que puede alcanzar hasta $15 \mathrm{~m}$ de altura, quien aparte de tener hojas que pierden menor cantidad de agua durante la exposición a temperatura igual a $40^{\circ} \mathrm{C}$, también emplea como estrategia, la perdida de sus hojas después de una sequía prolongada (Zapata, 2010).

Se hace indispensable incrementar los estudios asociados a la evaluación del comportamiento ecosfisiológico de las plantas locales, complementados con metodologías orientadas al conocimiento de aspectos histológicos o ultraestructurales que permitan identificar con mayor grado de precisión, aquellos atributos indispensables para que las plantas seleccionadas para los procesos de restauración ecológica y ambiental de los ecosistemas locales puedan ser las más idóneas; estos estudios además pueden enriquecerse con el conocimiento de la expresión génica o indicadores de estrés hídrico como el $\mathrm{H}_{2} \mathrm{O}_{2}$ (Begcy, 2011), la densidad estomática (González-Bermúdez, 2013) y ensayos in situ y ex situ, para verificar respuestas de las especies locales y las introducidas (González, 2001).

\section{CONCLUSIONES}

Existen once especies de plantas en los ecosistemas estratégicos estudiados, que se caracterizan por su heterogeneidad en cuanto a la tasa de deshidratación, lo cual está relacionado con el alto grado de intervención de estos ecosistemas, lo cual repercute en la funcionalidad y 
mantenimiento de los servicios ecosistémicos que se derivan de estos. La aplicación de estudios orientados a conocer el grado de perdida de agua por parte de plantas presentes en ecosistemas terrestres locales, resultan de gran utilidad para una adecuada gestión de los ecosistemas terrestres, ya que con ello se pueden identificar aquellas especies que representan mayor garantía de adaptación a las alteraciones que actualmente induce el fenómeno del cambio climático.

AGRADECIMIENTOS Los autores expresan sus agradecimientos a la Fundación Universitaria Tecnológico Comfenalco por habilitar las instalaciones de los laboratorios de ciencias básicas y aplicadas de la facultad de ingeniería para el desarrollo de los análisis de las muestras recolectadas en el área de estudio.

\section{ReFERENCIAS}

Afanador, F. O. (2008). Ortofotografía digital y datos LIDAR para el control y administración del litoral en la Isla de Tierra Bomba, Caribe Colombiano. Boletín Científico CIOH No. 26.

Alcaldía Mayor de Cartagena de Indias, Decreto 0977. (2001). Plan de Ordenamiento Territorial (POT), del Distrito Turístico y Cultural de Cartagena de Indias. Cartagena, Colombia..

Bartoskova H., J. Komenda \& J. Naus. (1999). Functional changes of photosystem II in the moss Rhizomnium punctatum (Hedw.) Induced by diffrent rates of dark desication. J. Plant Physiol., 54, 597-604.

Basnayake, J. C. (1996). Influence of rate of development of water deficit on the expression of maximum osmotic adjustment and desiccation tolerance in three grain sorghum lines. Filed Crops Research, 49, 65-76.

Begcy K., E. Mariano, L. Mantiello, A. Nunes, P. Mazzafera, I. Maia \& M. Menosi. (2011). An Arabidopsis Mitochondrial Uncoupling Protein Confers Tolerance to Drought and Salt Stress in Transgenic Tobacco Plants. PLoS ONE., 6(8), 1-9. doi:10.1371/journal.pone.0023776

Camia, A. B. (1999). Meteorological fire danger indices and remote sensing. Remote Sensing of Large Wildfires, 39-59.

Chuvieco, E. S. (2001). Estimación del estado hídrico de la vegetación a partir de sensores de alta y baja resolución. Revista Internacional de ciencia y tecnología, 1, 1-16.

Cocero D., D. Riaño \& E. Chuvieco. (2000). Estimación de la humedad de diferentes especies vegetales mediterráneas mediante distintos sensores de teledetección. Tecnologías Geográficas para el Desarrollo Sostenible. Departamento de Geografía. Universidad de Alcalá. 200-221.
Drennan, P. M. (2009). Marginal and laminar hydathodelike structures in the leaves of the desiccation-tolerant angiosperm Myrothamnus flabellifolius Welw. Flora Morfology, Distribution, Functional Ecology of Plants, 204(3), 210-219.

Garcés F. \& C. Forcellini. (2011). Peso de hojas como herramienta para estimar el área foliar en Soya. Ciencia y Tecnología, 4(1), 13-18.

González, H. (2001). Adaptación a la sequía de la planta arbustiva de matorral Espinoso Tamaulipecco. Ciencia UANL., 4, 454-461.

González-Bermúdez, A. (2013). Respuesta de la densidad estomática a características foliares e individuales en tres especies de árboles en Gamboa , Panamá. Pensamiento Actual, Vol. 13 (20), 43-49.

Jones R. (1995). Sucrose prevents foliage dessication in cut Leucadendron "Silvan Red" during cool storage. Postharvest Biology and Technology, 6(3), 293-301.

Hegbe J. \& M. Hossein. (2010). Water relations, gas Exchange, and yield of processing tomato under reduced irrigation. Revista Mexicana de Ciencias Agrícolas, 1(3), 445-450.

Ministerio de Ambiente y Desarrollo Sostenible (Minambiente). Recuperadoel02de05de2014,dehttp://www.minambiente. gov.co/documentos/DocumentosBiodiversidad/ proyectos_norma/proyectos/241011_proy_dec_ley_2811_ ordenacion_forestal_121111.pdf.

Oliver M., R. Jain., T. Balbuena, G. Agrawal., F. Gasulla \& J. Thelen. (2011). Phitochemistry, 72, 1273-1284.

Pence V., S. Dunford \& S. Redella. (2005). Differential effcts of abscisic acido on desecation tolerance and carbohydrates in three species of liveworts. Journal of Plant Physiology, $162,1331-1337$.

Rodríguez-Pérez, L. (2006). Implicaciones fisiológicas de la osmoregulación en plantas. Agronomía Colombiana, 24(19), 28-37.

Zapata, P. (2010). Efecto del guácimo (Guazuma ulmifolia), carao (Cassia grandis) y roble (Tabebuia rosea) sobre la productividad primaria neta aérea y composición florística de pasturas naturales en Muy Muy y Matiguás, Nicaragua. (Tesis de magíster). Recuperado de http://orton.catie.ac.cr/repdoc/A4665E/A4665E.PDF 